\title{
INTERSECTIONS OF RECURRENCE SEQUENCES
}

\author{
MICHAEL A. BENNETT AND ÁKOS PINTÉR \\ (Communicated by Matthew Papanikolas)
}

\begin{abstract}
We derive sharp upper bounds for the size of the intersection of certain linear recurrence sequences. As a consequence of these, we partially resolve a conjecture of Yuan on simultaneous Pellian equations, under the condition that one of the parameters involved is suitably large.
\end{abstract}

\section{INTRODUCTION}

Let $\left\{u_{m}\right\}_{m=0}^{\infty}$ and $\left\{v_{n}\right\}_{n=0}^{\infty}$ be integral linear recurrence sequences. That is, let us suppose that there exist positive integers $h$ and $k$, and rational integers $a_{1}, a_{2}, \ldots, a_{h}, u_{0}, u_{1}, \ldots u_{h-1}, b_{1}, b_{2}, \ldots, b_{k}$ and $v_{0}, v_{1}, \ldots v_{k-1}$, such that

$$
u_{m+h}=a_{h-1} u_{m+h-1}+a_{h-2} u_{m+h-2}+\cdots+a_{0} u_{m}, \text { for } m=0,1,2, \ldots
$$

and

$$
v_{n+k}=b_{k-1} v_{n+k-1}+b_{k-2} v_{n+k-2}+\cdots+b_{0} v_{n}, \text { for } n=0,1,2, \ldots
$$

Then, as is well-known, there further exist algebraic integers

$$
\alpha_{1}, \ldots, \alpha_{h}, \beta_{1}, \ldots, \beta_{k}
$$

and polynomials $P_{1}, \ldots, P_{h}, Q_{1}, \ldots, Q_{k}$, with algebraic coefficients, such that we may write

$$
u_{m}=P_{1}(m) \alpha_{1}^{m}+\cdots+P_{h}(m) \alpha_{h}^{m}, \quad P_{1} \neq 0
$$

and

$$
v_{n}=Q_{1}(n) \beta_{1}^{n}+\cdots+Q_{k}(n) \beta_{k}^{n}, Q_{1} \neq 0
$$

for each pair of nonnegative integers $m$ and $n$. It is certainly possible that such sequences may share values, even infinitely many, but, typically, our expectation is that their intersection is finite. Our goal in this paper is to derive a very sharp estimate for the size of such an intersection, for a specific class of recurrences. This problem is studied in detail in much greater generality by, for example, Laurent [8] and Schlickewei and Schmidt [13, 14.

Received by the editors October 1, 2013 and, in revised form, January 19, 2014.

2010 Mathematics Subject Classification. Primary 11J86, 11B39, 11 D61.

Key words and phrases. Recurrence sequences, simultaneous linear forms in logarithms.

The first author was supported in part by a grant from NSERC.

The second author was supported in part by the Hungarian Academy of Sciences, OTKA grants K100339, NK101680, NK104208 and by the European Union and the European Social Fund through project Supercomputer, the national virtual lab (grant no.: TÁMOP-4.2.2.C11/1/KONV-2012-0010). 
As a qualitative example of the type of theorem available in the literature, let us mention the main result of [11]:

Theorem 1.1. (Mignotte) For $\left\{u_{m}\right\}_{m=0}^{\infty}$ and $\left\{v_{n}\right\}_{n=0}^{\infty}$ as above with the additional assumptions that

$$
\left|\alpha_{1}\right|>\max \left\{1,\left|\alpha_{2}\right|, \ldots,\left|\alpha_{h}\right|\right\} \text { and }\left|\beta_{1}\right|>\max \left\{1,\left|\beta_{2}\right|, \ldots,\left|\beta_{k}\right|\right\},
$$

there exists an effectively computable constant $m_{0}$ such that if $u_{m}=v_{n}$ with $m \geq$ $m_{0}$, then necessarily

$$
P_{1}(m) \alpha_{1}^{m}=Q_{1}(n) \beta_{1}^{n} .
$$

If this last relation occurs infinitely often, then there exist positive integers $x$ and $y$ such that $\alpha_{1}^{x}=\beta_{1}^{y}$. If, further, the polynomials $P_{1}$ and $Q_{1}$ are actually constant, then the set of pairs of integers $(m, n)$ for which $u_{m}=v_{n}$ lie in the union of a finite set with a finite number of arithmetic progressions.

If we can rule out the presence of such progressions, then the corresponding intersection is necessarily finite. In this context, our main result quantifies the size of such an intersection, at least under favourable circumstances. While our arguments lead to a more general statement, we will restrict our attention somewhat in the interests of simplicity. Here and henceforth, by $h(\alpha)$ we mean the absolute logarithmic Weil height of an algebraic number of degree $d$, given by the formula

$$
h(\alpha)=\frac{1}{d}\left(\log \left|a_{0}\right|+\sum_{i=1}^{d} \log \max \left(1,\left|\alpha^{(i)}\right|\right)\right),
$$

where $a_{0}$ is the leading coefficient of the minimal polynomial of $\alpha$ over $\mathbb{Z}$ and the $\alpha^{(i)} \mathrm{s}$ are the conjugates of $\alpha$ in the field of complex numbers.

Theorem 1.2. Suppose that $\left\{u_{m}\right\}_{m=0}^{\infty}$ and $\left\{v_{n}\right\}_{n=0}^{\infty}$ are integral linear recurrence sequences, that

$$
\alpha_{1}, \ldots, \alpha_{h}, \beta_{1}, \ldots, \beta_{k}
$$

are algebraic integers, and that

$$
P_{1}, \ldots, P_{h}, Q_{1}, \ldots, Q_{k}
$$

are algebraic numbers, for which

$$
u_{m}=P_{1} \alpha_{1}^{m}+\cdots+P_{h} \alpha_{h}^{m}, \quad P_{1} \neq 0,
$$

and

$$
v_{n}=Q_{1} \beta_{1}^{n}+\cdots+Q_{k} \beta_{k}^{n}, Q_{1} \neq 0
$$

hold, and we have

$$
\left|\alpha_{1}\right|>\max \left\{1,\left|\alpha_{2}\right|, \ldots,\left|\alpha_{h}\right|\right\} \text { and }\left|\beta_{1}\right|>\max \left\{1,\left|\beta_{2}\right|, \ldots,\left|\beta_{k}\right|\right\} \text {. }
$$

Let us assume further that $\alpha_{1}, \beta_{1}, P_{1}$ and $Q_{1}$ are real, that $\alpha_{1}$ and $\beta_{1}$ are multiplicatively independent and that $P_{1} \neq Q_{1}$. Defining

$$
M=\max \left\{h\left(P_{i}\right), h\left(Q_{j}\right): 1 \leq i \leq h, 1 \leq j \leq k\right\}
$$

and

$$
N=\max \left\{h, k, M, \log \left|\beta_{1}\right|, 3\right\},
$$

there exists an effectively computable absolute constant $C$ such that if

$$
\log \left|\alpha_{1}\right| \geq C M \log \left|\beta_{1}\right| \log ^{3} N
$$


then there is at most one pair of positive integers $(m, n)$ with

$$
u_{m}=v_{n} \quad \text { and } \quad P_{1} \alpha_{1}^{m} \neq Q_{1} \beta_{1}^{n} .
$$

It is worth observing that the dominant root condition (1.3) is one that occurs somewhat naturally in a variety of contexts in the theory of recurrence sequences.

In the case where the two recurrences under consideration are both binary, there are many results in the literature establishing absolute bounds upon the size of their intersections, under various restrictions. One of the simplest cases is that of simultaneous Pellian equations, where, given distinct nonsquare positive integers $a$ and $b$, we find that the number of positive integral triples $(x, y, z)$ satisfying

$$
x^{2}-a z^{2}=1, \quad y^{2}-b z^{2}=1
$$

is at most two (a bound that is achieved for infinitely many pairs $(a, b)$; see [2] and [17]). In the case of the similar simultaneous equations

$$
x^{2}-a y^{2}=1, y^{2}-b z^{2}=1,
$$

the number of positive solutions has also been shown (see [6] and [7]) to be at most two. In this situation, however, we know of no pair $(a, b)$ for which two such solutions actually exist and Yuan (Conjecture 1.1 of [18]) suggests that (1.6) has, in fact, at most a single positive solution $(x, y, z)$ for a fixed pair $(a, b)$. We can verify this conjecture (in a rather stronger form), provided $b$ is sufficiently large as a function of $a$. Indeed, a somewhat straightforward corollary of Theorem 1.2 in this case is the following

Corollary 1.3. Let $a$ and $b$ be nonsquare positive integers and let $\varepsilon_{a}$ and $\varepsilon_{b}$ denote the fundamental units in $\mathbb{Q}(\sqrt{a})$ and $\mathbb{Q}(\sqrt{b})$, respectively. Then there exists an effectively computable absolute constant $\kappa$ such that if

$$
\log \varepsilon_{b}>\kappa \log a \log \varepsilon_{a}\left(\log \max \left\{\log \varepsilon_{a}, 3\right\}\right)^{3},
$$

the system of simultaneous equations

$$
\left|x^{2}-a y^{2}\right|=\left|y^{2}-b z^{2}\right|=1
$$

has at most one solution in positive integers $x, y$ and $z$.

It is easy to observe that this result is sharp. Defining

$$
T_{k}=\frac{(3+2 \sqrt{2})^{k}-(3-2 \sqrt{2})^{k}}{2 \sqrt{2}},
$$

if we choose $(a, b)=\left(2, T_{k}^{2}-1\right)$ for $k$ suitably large, then inequality (1.7) is satisfied and equations (1.8) have the positive integer solution $(x, y, z)=\left(U_{k}, T_{k}, 1\right)$, where

$$
U_{k}=\frac{(3+2 \sqrt{2})^{k}+(3-2 \sqrt{2})^{k}}{2} .
$$

In what follows, our principal tool will be lower bounds for linear forms in complex logarithms of algebraic numbers. Our hope is that this paper will serve as a small advertisement for the theory of simultaneous linear forms in logarithms (indeed, Theorem 1.2 depends fundamentally upon such estimates). These results have been around for many years, dating to the early days of development of the general theory, but are neither widely known, nor widely used. One has the sense that they could find application rather more broadly than is currently the case. Interested readers are directed to [3], 4], [5], 9], 12, [15] and [16]. 


\section{Proof of Theorem 1.2}

We begin by proving Theorem 1.2 . Suppose that we have

$$
u_{m_{1}}=v_{n_{1}} \text { and } u_{m_{2}}=v_{n_{2}},
$$

where $\left(m_{1}, n_{1}\right)$ and $\left(m_{2}, n_{2}\right)$ are distinct pairs of positive integers. Suppose further that

$$
P_{1} \alpha_{1}^{m_{1}} \neq Q_{1} \beta_{1}^{n_{1}} \text { and } P_{1} \alpha_{1}^{m_{2}} \neq Q_{1} \beta_{1}^{n_{2}}
$$

Define

$$
\delta=\frac{1}{2} \min \left\{1-\max _{2 \leq i \leq h}\left\{\frac{\log \left|\alpha_{i}\right|}{\log \left|\alpha_{1}\right|}\right\}, 1-\max _{2 \leq j \leq k}\left\{\frac{\log \left|\beta_{j}\right|}{\log \left|\beta_{1}\right|}\right\}\right\} .
$$

Then, assuming (1.4) for suitably large $C$, it follows from (1.3) that

$$
\left|P_{1} \alpha_{1}^{m_{i}}-Q_{1} \beta_{1}^{n_{i}}\right|<\frac{1}{2} \min \left\{\left|P_{1} \alpha_{1}^{m_{i}}\right|,\left|Q_{1} \beta_{1}^{n_{i}}\right|\right\}^{1-\delta} .
$$

Since $P_{1}, Q_{1}, \alpha_{1}$ and $\beta_{1}$ are real, if we consider the linear forms

$$
\Lambda_{i}=m_{i} \log \left|\alpha_{1}\right|-n_{i} \log \left|\beta_{1}\right|+\log \left|P_{1} / Q_{1}\right|, \quad i=1,2,
$$

we therefore have that

$$
\log \left|\Lambda_{i}\right|<-\delta \min \left\{\log \left|P_{1} \alpha_{1}^{m_{i}}\right|, \log \left|Q_{1} \beta_{1}^{n_{i}}\right|\right\} .
$$

Assumption (2.1) ensures further that $\Lambda_{i} \neq 0$. Since the $\alpha_{i}$ and the $\beta_{j}$ are roots of the companion polynomials of the recurrences defining $\left\{u_{m}\right\}_{m=0}^{\infty}$ and $\left\{v_{n}\right\}_{n=0}^{\infty}$, respectively, monic polynomials with integer coefficients, it follows from (1.3) that

$$
h\left(\alpha_{1}\right) \leq \log \left|\alpha_{1}\right| \text { and } h\left(\beta_{1}\right) \leq \log \left|\beta_{1}\right| .
$$

We appeal to standard bounds for linear forms in logarithms to derive a lower bound upon $\left|\Lambda_{i}\right|$. Specifically, we use the main result of [1].

Theorem 2.1. (Baker-Wüstholz) Let $\alpha_{1}, \ldots, \alpha_{n}$ be algebraic numbers different from 0 and 1 , in a fixed number field $\mathbb{K}$ of degree $d$. Define the modified height $h^{\prime}$ by

$$
h^{\prime}(\alpha)=\max \left\{h(\alpha), \frac{|\log \alpha|}{d}, \frac{1}{d}\right\}
$$

for every nonzero $\alpha$ in $\mathbb{K}$, where $h(\alpha)$ is the usual logarithmic Weil height. Let $b_{1}, \ldots, b_{n}$ be rational integers, not all 0 , and with absolute values less than $B \geq 3$. Setting

$$
\Lambda=b_{1} \log \alpha_{1}+\ldots+b_{n} \log \alpha_{n} \neq 0,
$$

we have

$$
\log |\Lambda|>-C(n, d) \cdot h^{\prime}\left(\alpha_{1}\right) \cdots h^{\prime}\left(\alpha_{n}\right) \log B,
$$

with

$$
C(n, d)=18(n+1) ! n^{n+1}(32 d)^{n+2} \log (2 n d) .
$$

We will also have need of a simultaneous analogue of this result, due to Loxton [9], which provides a sharper lower bound for linear combinations of logarithms of algebraic numbers. 
Theorem 2.2. (Loxton) Set

$$
\Lambda_{i}=b_{i 1} \log \alpha_{1}+\ldots+b_{i n} \log \alpha_{n},(1 \leq i \leq t),
$$

where $\alpha_{1}, \ldots, \alpha_{n}$ are multiplicatively independent elements of a fixed number field $\mathbb{K}$ of degree $d$, the matrix of rational integers $\left(b_{i j}\right)$ has rank $t$ and the $\log \alpha_{j}$ are the principal values. Let $A_{j} \geq 4$ be an upper bound for $\exp \left(h\left(\alpha_{j}\right)\right), B \geq 4$ be an upper bound for $\max \left\{\left|b_{i j}\right|\right\}$ and put $\Omega=\log A_{1} \cdots \log A_{n}$. Then

$$
\max _{1 \leq i \leq t}\left|\Lambda_{i}\right|>\exp \left\{-\mathfrak{C}(\Omega \log \Omega)^{1 / t} \log (B \Omega)\right\} \text { with } \mathfrak{C}=(16 n d)^{200 n} .
$$

Applying Theorem 2.1 thus yields a lower bound of the shape

$$
\log \left|\Lambda_{i}\right| \gg-M \log n_{i} \log \left|\beta_{1}\right| \log \left|\alpha_{1}\right|,
$$

whereby we reach the conclusion that

$$
\frac{n_{i}}{\log n_{i}} \ll M \log \left|\alpha_{1}\right|
$$

and hence, once again appealing to (1.4),

$$
n_{i} \ll M \log \left|\alpha_{1}\right| \log \log \left|\alpha_{1}\right| .
$$

Next, applying Theorem 2.2 (which we may do since we assume that $P_{1} \neq Q_{1}$ and that $\alpha_{1}$ and $\beta_{1}$ are multiplicatively independent) and writing

$$
\Omega=M \log \left|\alpha_{1}\right| \log \left|\beta_{1}\right|
$$

we obtain the inequality

$$
\max \left\{\log \left|\Lambda_{1}\right|, \log \left|\Lambda_{2}\right|\right\} \gg-(\Omega \log \Omega)^{1 / 2} \log \max \left\{n_{1} \Omega, n_{2} \Omega\right\} .
$$

From (2.3), it follows that

$$
\max \left\{\log \left|\Lambda_{1}\right|, \log \left|\Lambda_{2}\right|\right\} \gg-\Omega^{1 / 2} \log ^{3 / 2} \Omega .
$$

Combining this with inequality (2.2), we therefore have

$$
\log \left|\alpha_{1}\right| \ll \Omega^{1 / 2} \log ^{3 / 2} \Omega,
$$

whence

$$
\log \left|\alpha_{1}\right| \ll M \log \left|\beta_{1}\right| \log ^{3} \Omega,
$$

contradicting (1.4) if the constant $C$ is chosen suitably large. This completes the proof of Theorem 1.2 .

\section{Proof of Corollary 1.3}

We next turn our attention to Corollary 1.3. If we have two solutions in positive integers to equation (1.8), say $\left(x_{1}, y_{1}, z_{1}\right)$ and $\left(x_{2}, y_{2}, z_{2}\right)$, with $x_{1}<x_{2}$, then

$$
y_{i}=\frac{\varepsilon_{a}^{n_{i}}-\overline{\varepsilon_{a}} n_{i}}{2 \sqrt{a}}=\frac{\varepsilon_{b}^{m_{i}}+\overline{\varepsilon_{b}} m_{i}}{2}, \text { for } i \in\{1,2\}
$$

where the $n_{i}$ and $m_{i}$ are positive integers and $\overline{\varepsilon_{a}}$ and $\overline{\varepsilon_{b}}$ are the conjugates of $\varepsilon_{a}$ and $\varepsilon_{b}$ in $\mathbb{Q}(\sqrt{a})$ and $\mathbb{Q}(\sqrt{b})$, respectively. That is, we have $u_{m_{i}}=v_{n_{i}}$ for $i=1,2$, where the recurrences satisfy $h=k=2$,

$$
\begin{gathered}
\alpha_{1}=\varepsilon_{b}, \alpha_{2}=\overline{\varepsilon_{b}}, \beta_{1}=\varepsilon_{a}, \beta_{2}=\overline{\varepsilon_{a}} \\
P_{1}=P_{2}=1 / 2, Q_{1}=\frac{1}{2 \sqrt{a}} \text { and } Q_{2}=-\frac{1}{2 \sqrt{a}} .
\end{gathered}
$$


If we have

$$
P_{1} \alpha_{1}^{m_{i}}=Q_{1} \beta_{1}^{n_{i}}
$$

for either $i=1$ or 2 , then (3.1) implies that

$$
\varepsilon_{a}^{n_{i}}=\sqrt{a} \varepsilon_{b}^{m_{i}} \text { and }-{\overline{\varepsilon_{a}}}^{n_{i}}=\sqrt{a}{\overline{\varepsilon_{b}}}^{m_{i}},
$$

whence $a= \pm 1$, an immediate contradiction. Since $\left|\overline{\varepsilon_{a}}\right|=\left|\varepsilon_{a}\right|^{-1}$ and $\left|\overline{\varepsilon_{b}}\right|=\left|\varepsilon_{b}\right|^{-1}$, these recurrences satisfy (1.3) and hence, applying Theorem 2.2. we conclude as stated, at least provided the fundamental units $\varepsilon_{a}$ and $\varepsilon_{b}$ are multiplicatively independent.

Let us now suppose that $\varepsilon_{a}$ and $\varepsilon_{b}$ are multiplicatively dependent, satisfying, say,

$$
\varepsilon_{a}^{r}=\varepsilon_{b}^{s},
$$

for $r$ and $s$ coprime, positive integers. If we have even a single solution to equation (1.8) in positive integers $(x, y, z)$, then there exist positive integers $m$ and $n$ such that

$$
y=\frac{\varepsilon_{a}^{n}-{\overline{\varepsilon_{a}}}^{n}}{2 \sqrt{a}}=\frac{\varepsilon_{b}^{m}+{\overline{\varepsilon_{b}}}^{m}}{2} .
$$

The corresponding linear form

$$
\Lambda=m \log \varepsilon_{b}-n \log \varepsilon_{a}+\log (\sqrt{a})
$$

can be rewritten as

$$
\Lambda=\left(\frac{m r}{s}-n\right) \log \varepsilon_{a}+\log (\sqrt{a})
$$

Since we have

$$
|\Lambda|=\log \left|{\overline{\varepsilon_{a}}}^{n}+\sqrt{a}{\overline{\varepsilon_{b}}}^{m}\right| \ll \sqrt{a} \varepsilon_{b}^{-m} \ll a \varepsilon_{a}^{-n},
$$

it follows that

$$
\log |\Lambda| \ll \log a-n \log \varepsilon_{a} .
$$

In the other direction, let us begin by noting that $m r \geq n s$ implies the inequality $|\Lambda| \geq \log (\sqrt{a})$, contradicting (1.7) and (3.3). We may thus assume that $\max \{m r, n s\}=n s$. Applying part $\mathrm{C}$ of Theorem 3 of Loxton and van der Poorten [10], we have that

$$
s \leq \frac{2}{\log \left(\frac{1}{2}(1+\sqrt{5})\right)} \log \left(\varepsilon_{a}\right),
$$

whence, from Theorem 2.1.

$$
\log |\Lambda| \gg-\log \varepsilon_{a} \log a \log (n s)
$$

and so, appealing to (3.3) and (3.4),

$$
n \ll \log a \log (n s) \ll \log a \log \left(n \log \left(\varepsilon_{a}\right)\right) .
$$

Combining this with (3.2), which implies that

$$
\log \left(\varepsilon_{b}\right) \ll n \log \left(\varepsilon_{a}\right),
$$

we contradict (1.7), provided $\kappa$ is chosen to be suitably large. This completes the proof of Corollary 1.3 .

\section{ACKNOWLEDGEMENTS}

The authors would like to thank Yann Bugeaud and the anonymous referee for bringing our attention to incorrect statements in an earlier version of this work. 


\section{REFERENCES}

[1] A. Baker and G. Wüstholz, Logarithmic forms and group varieties, J. Reine Angew. Math. 442 (1993), 19-62, DOI 10.1515/crll.1993.442.19. MR 1234835 (94i:11050)

[2] Michael A. Bennett, Mihai Cipu, Maurice Mignotte, and Ryotaro Okazaki, On the number of solutions of simultaneous Pell equations. II, Acta Arith. 122 (2006), no. 4, 407-417, DOI 10.4064/aa122-4-4. MR2234424(2007e:11040)

[3] B. Brindza, Thue equations and multiplicative independence, Number theory and cryptography (Sydney, 1989), London Math. Soc. Lecture Note Ser., vol. 154, Cambridge Univ. Press, Cambridge, 1990, pp. 213-220. MR1055412 (91h:11061)

[4] B. Brindza and K. Győry, On unit equations with rational coefficients, Acta Arith. 53 (1990), no. 4, 367-388. MR1067769 (91h:11020)

[5] Yann Bugeaud, On the greatest prime factor of $(a b+1)(b c+1)(c a+1)$, Acta Arith. 86 (1998), no. 1, 45-49. MR.1642613 (99k:11141)

[6] Mihai Cipu and Maurice Mignotte, On the number of solutions to systems of Pell equations, J. Number Theory 125 (2007), no. 2, 356-392, DOI 10.1016/j.jnt.2006.09.016. MR2332594 (2009b:11054)

[7] Bo He, The number of solutions to the simultaneous Pell equations $x^{2}-a y^{2}=1$ and $y^{2}-$ $b z^{2}=1$ (Chinese, with English and Chinese summaries), Acta Math. Sinica (Chin. Ser.) 51 (2008), no. 4, 721-726. MR2454009 (2009j:11048)

[8] Michel Laurent, Équations exponentielles polynômes et suites récurrentes linéaires (French), Astérisque 147-148 (1987), 121-139, 343-344. Journées arithmétiques de Besançon (Besançon, 1985). MR891424 (88h:11011)

[9] J. H. Loxton, Some problems involving powers of integers, Acta Arith. 46 (1986), no. 2, 113-123. MR842939 (87j:11071)

[10] J. H. Loxton and A. J. van der Poorten, Multiplicative dependence in number fields, Acta Arith. 42 (1983), no. 3, 291-302. MR.729738(86b:11052)

[11] M. Mignotte, Intersection des images de certaines suites récurrentes linéaires (French), Theoret. Comput. Sci. 7 (1978), no. 1, 117-122. MR0498356 (58 \#16486)

[12] K. Ramachandra, A note on Baker's method, J. Austral. Math. Soc. 10 (1969), 197-203. MR0246825 (40 \#94)

[13] H. P. Schlickewei and W. M. Schmidt, Linear equations in members of recurrence sequences, Ann. Scuola Norm. Sup. Pisa Cl. Sci. (4) 20 (1993), no. 2, 219-246. MR1233637(94h:11004)

[14] Hans Peter Schlickewei and Wolfgang M. Schmidt, The intersection of recurrence sequences, Acta Arith. 72 (1995), no. 1, 1-44. MR.1346803 (96g:11011)

[15] C. L. Stewart, On sets of integers whose shifted products are powers, J. Combin. Theory Ser. A 115 (2008), no. 4, 662-673, DOI 10.1016/j.jcta.2007.07.010. MR2407918(2009a:11059)

[16] Michel Waldschmidt, Simultaneous approximation of logarithms of algebraic numbers, Colloque Franco-Japonais: Théorie des Nombres Transcendants (Tokyo, 1998), Sem. Math. Sci., vol. 27, Keio Univ., Yokohama, 1999, pp. 123-134. MR.1726530 (2000m:11062)

[17] Pingzhi Yuan, On the number of solutions of simultaneous Pell equations, Acta Arith. 101 (2002), no. 3, 215-221, DOI 10.4064/aa101-3-2. MR1875840 (2003e:11034)

[18] Pingzhi Yuan, On the number of solutions of $x^{2}-4 m(m+1) y^{2}=y^{2}-b z^{2}=1$, Proc. Amer. Math. Soc. 132 (2004), no. 6, 1561-1566, DOI 10.1090/S0002-9939-04-07418-0. MR.2051114 (2004m:11043)

Department of Mathematics, University of British Columbia, Vancouver, BC Canada V6T $1 \mathrm{Z} 2$

E-mail address: bennett@math.ubc.ca

Institute of Mathematics, MTA-DE Research Group "Equations, Functions and Curves", Hungarian Academy of Sciences and University of Debrecen, P.O. Box 12, H-4010 Debrecen, Hungary

E-mail address: apinter@science.unideb.hu 